\title{
Left Ventricular Hypertrophy and Asymptomatic Cardiac Function Impairment in Chinese Patients with Simple Obesity using Echocardiography
}

\author{
Ting Sun ${ }^{a}$ Jing Xie ${ }^{b} \quad$ Lili Zhu $^{a, c}$ Zhihua Han ${ }^{a} \quad$ Yushui Xie ${ }^{a}$ \\ a Department of Cardiology, Shanghai Ninth People's Hospital, Shanghai JiaoTong University \\ School of Medicine, Shanghai, P.R. China; ${ }^{b}$ Department of Cardiology, Cashi Second \\ People's Hospital, Cashi, Xinjiang, P.R. China; 'The Fourth Affiliated Hospital of Anhui \\ Medical University, Hefei, Anhui, P.R. China
}

\author{
Key Words \\ Obesity · Body mass index · Echocardiography · Tissue Doppler imaging · Left ventricular \\ function
}

\begin{abstract}
Background: Simple obesity in China is rising rapidly and causing increasing concern. The objectives of our study are to investigate cardiac structure and function in individuals with simple obesity and to analyze the effect of BMI on left ventricular structure and function. Methods: Between January 2012 and July 2014, echocardiography was performed in 361 consecutive patients visiting the outpatient echocardiography center for a health examination or cardiac evaluation before a weight loss operation in our hospital. Echocardiographic indices, waist-to-hip ratios, BMI, and metabolic markers were evaluated. We analyzed these data using Student's t test (normally distributed) or a nonparametric test (not normally distributed) for continuous variables and chi-square test for categorical variables. Multivariate correlation and regression analysis were conducted for comparisons. Results: The study sample was divided into three groups: a normal/overweight group (BMI $<28.0 \mathrm{~kg} / \mathrm{m}^{2}$ ), a mildly/moderately obese group (BMI $28-39.9 \mathrm{~kg} / \mathrm{m}^{2}$ ), and a severely obese group (BMI $\geq 40 \mathrm{~kg} / \mathrm{m}^{2}$ ). There were no significant differences in clinical and laboratory characteristics among the groups, except for BMI and waist-to-hip ratio. The severely obese group had a higher left ventricular end diastolic diameter (LVEDD; $p<0.01$ ) and lower left ventricular ejection fraction (LVEF; $p<0.01$ ) than the mildly/moderately obese group, which had a higher LVEDD and LV mass index (LVMI) than the normal/overweight group. BMI correlated well with LVEDD, left ventricular posterior wall thickness at end-diastole (LVPW), LV mass, LVMI, and E/e'. In addition, age was significantly associated with some echocardiographic parameters, including left atrial dimension
\end{abstract}


$(r=0.366, p<0.01)$, LVPW $(r=0.347, p<0.01)$, interventricular septal thickness at enddiastole $(r=0.351, p<0.01)$, and $E / A(r=-0.47, p<0.01)$. Conclusions: Simple obesity caused cardiac structural changes, including LV hypertrophy and LV enlargement, and severe obesity resulted in asymptomatic LV systolic and diastolic function impairment.

(C) 2015 S. Karger GmbH, Freiburg

\section{Introduction}

Obesity, which is characterized by excessive fat deposition, is a growing problem in China because of economic development and improved living standards [1-3]. Based on its pathogenesis, obesity is separated into simple obesity and severe obesity [4]. Simple obesity is the presence of obesity without other diseases, often caused by unhealthy lifestyles, including poor dietary habits and limited physical activity [4]. Severe obesity rapidly shortens life expectancy and increases the risk of chronic and fatal diseases. Many studies have shown that severe obesity is associated with significant cardiac abnormalities, including abnormal left ventricular (LV) geometry and function [5]. However, these studies focused on obesity in patients with metabolic syndrome or associated disorders such as diabetes [6], hypertension [6, 7], hyperlipidemia [6, 8], and adipose inflammation [9]. Limited information is available on the relationship between simple obesity and cardiac structure or function. The aims of our study were to investigate echocardiographic characteristics, blood pressure, waist-to-hip ratio (WHR), and metabolic markers in a population with simple obesity and to analyze the effect of BMI on cardiac structure and function and the relationships between BMI and other clinical features.

\section{Patients and Methods}

\section{Study Population}

Between January 2012 and July 2014, we evaluated 361 consecutive patients who visited the outpatient echocardiography center for a health examination or cardiac evaluation before a weight loss operation in our hospital. Patients were eligible for inclusion if they fulfilled the following criteria: age > 18 years, sinus rhythm, healthy, and a signed consent form. Exclusion criteria were as follows: hyperlipidemia; hyperthyroidism; diabetes; hypertension; acute inflammatory disorders; ischemia; trauma; surgery; pancreatitis; febrile disorders; connective tissue disorder; kidney disease; lung disease; and primary and secondary heart diseases, including heart failure, coronary heart disease, segmental wall motion abnormalities, restrictive pericarditis, valvular heart disease, arrhythmia, cardiomyopathy, infective endocarditis, and cardiac tumors. Patients were excluded if they had a history of taking cardiac medication. Patients were also excluded if their ultrasonic signal was very poor. All patients had a medical record completed, including age, gender, height, weight, and abdominal and hip circumferences. Written informed consent was obtained from all study participants, and the study protocol was approved by the Shanghai Ninth People's Hospital Ethics Committee and Research Board.

\section{Measurement and Calculation}

Blood pressure (BP) was measured using a calibrated mercury sphygmomanometer in both arms while sitting after at least 30 min of rest, and the average of 3 recordings was used. Weight and height were measured with participants in light clothing and not wearing shoes. Abdominal and hip circumference measurements were performed by the same examiner with the patient in a standing position.

WHR was calculated as abdominal circumferences divided by hip circumferences. BMI was calculated as weight (in kilograms) divided by the square of height (in meters). Body surface area (BSA) was calculated using the DuBois formula as BSA $\left(\mathrm{m}^{2}\right)=$ weight $(\mathrm{kg})^{0.425} \times$ Height $(\mathrm{cm})^{0.725} \times 0.00718413$ [10].

\section{Laboratory Tests}

All of the subjects were investigated in the morning after an overnight fast. A venous cannula was inserted into the brachial vein for blood sampling. The blood samples were drawn into 5-ml EDTA glass 
Sun et al.: Left Ventricular Hypertrophy and Asymptomatic Cardiac Function

Impairment in Chinese Patients with Simple Obesity using Echocardiography

tubes. Serum was separated by centrifugation, and biochemical measurements were conducted immediately. Serum total cholesterol (Tchol), low-density lipoprotein cholesterol (LDL-C), high-density lipoprotein cholesterol (HDL-C), triglyceride (TG), creatinine (Cr) and blood glucose (BG) were assayed using routine laboratory techniques.

\section{Definitions and Groups}

Asian, European, and American people belong to different races; thus, the WHO's criteria are not very suitable for Chinese individuals. Based on Chinese obesity standards [11], the patients were characterized as normal weight if their BMI was between 18.5 and $23.9 \mathrm{~kg} / \mathrm{m}^{2}$, overweight if their BMI was between 24 and $27.9 \mathrm{~kg} / \mathrm{m}^{2}$, obese if their BMI was above $28 \mathrm{~kg} / \mathrm{m}^{2}$, and severely obese if their BMI was higher than $40 \mathrm{~kg} / \mathrm{m}^{2}$. We divided the study participants into three groups: Group 1, normal weight and overweight (G1, BMI $<28.0 \mathrm{~kg} / \mathrm{m}^{2}$ ); Group 2, mild and moderate obesity (G2, BMI 28-39.9 kg/m²); and Group 3, severe obesity (G3, BMI $\geq 40 \mathrm{~kg} / \mathrm{m}^{2}$ ).

\section{Echocardiographic Assessment}

Echocardiography, including standard two-dimensional M-mode, Doppler and tissue-Doppler measurements, was performed using the German Acuson Sequoia C512 ultrasound heartbeat graph examination instrument and a $3.25 \mathrm{MHz}$ transducer. All echo-Doppler studies were performed by the same observer, who was unaware of the clinical data, to avoid inter-reader variability. All measurements were averaged over three consecutive cardiac cycles. Special attention was focused on the location of the sample size as well as on gain, filter, and angulations, to obtain reliable measurements.

Left ventricular end diastolic diameter (LVEDD), left atrial dimension (LAD), interventricular septal thickness at end-diastole (IVS), and left ventricular posterior wall thickness at end-diastole (LVPW) were measured using the American Society of Echocardiography (ASE) and the European Society of Cardiology guidelines. Left ventricular (LV) mass was calculated according to the Devereux formula: $\mathrm{LV}$ mass $=1.04 \times$ $\left((\text { LVEDD + IVS + LVPW) })^{3}-(\text { LVEDD })^{3}\right)-13.6$ [12]. Thereafter, LV mass index (LVMI) was obtained by the following formula: $\mathrm{LV}$ mass / BSA $\left(\mathrm{g} / \mathrm{m}^{2}\right)[13]$.

LV systolic function was assessed by left ventricular ejection fraction (LVEF), which was measured using the modified Simpson's rule from two-dimensional echocardiographic tracings obtained in an apical fourchamber view. LV diastolic function was evaluated using the E/A and E/e' ratios, which were measured by pulsed-wave Doppler and pulsed-wave tissue Doppler imaging (TDI) [14]. Peak velocities of the early phase (E) and late phase (A) at the level of mitral valve leaflet tips were measured by pulsed-wave Doppler; then, the ratio (E/A) was calculated. TDI, guided by the two-dimensional four-chamber view, was performed to measure myocardial velocities. The setting was adjusted for a frame rate between 120 and $180 \mathrm{~Hz}$, and a cineloop of 3-5 consecutive heart beats was recorded [14]. Early diastolic ( $\left.\mathrm{e}^{\prime}\right)$ and late diastolic $\left(\mathrm{a}^{\prime}\right)$ myocardial velocities were measured from the average of septal and lateral tissue velocities in the apical four-chamber view, and then the $\mathrm{E} / \mathrm{e}^{\prime}$ ratio was calculated.

\section{Statistical Analysis}

The data were expressed as means \pm SD. Differences in the means of variables were evaluated using both parametric tests depending on the distribution of the variables. Categorical variables were analyzed using the chi-square test. Continuous variables were analyzed using Student's t-test (normally distributed) or a nonparametric test (not normally distributed) or analysis of variance followed by post-hoc analysis for multiple comparisons using Tukey's test to assess different subsets as appropriate. The associations between variables were studied using Pearson's correlation coefficient and forward stepwise multiple regression analysis. Statistical significance was considered as $\mathrm{p}<0.05$. All statistical analyses were performed using the Statistical Package for Social Sciences (SPSS for Windows, version 17.0; SPSS Inc., Chicago, IL, USA).

\section{Results}

\section{Clinical and Laboratory Characteristics}

Of the 361 participants (167 males, 194 females, mean age $34.2 \pm 10.7$ years, minimum 18 years, maximum 67 years), 74 participants had a normal BMI and 58 were overweight (G1), 67 were mildly obese and 50 were moderately obese (G2), and 112 were severely obese 
Table 1. Clinical and laboratory characteristics of studied patients
Table 2. Echocardiographic characteristics of studied patients
Sun et al.: Left Ventricular Hypertrophy and Asymptomatic Cardiac Function Impairment in Chinese Patients with Simple Obesity using Echocardiography

\begin{tabular}{llll}
\hline & G1 $(\mathrm{n}=132)$ & G2 $(\mathrm{n}=117)$ & $\mathrm{G} 3(\mathrm{n}=112)$ \\
\hline Age, years & $30.3 \pm 7.1$ & $34.4 \pm 10.8$ & $32.8 \pm 8.6$ \\
BMI, kg/m ${ }^{2}$ & $21.7 \pm 3.1$ & $32.4 \pm 3.6^{*}$ & $48.9 \pm 8.6^{*}$ \\
WHR & $0.84 \pm 0.07$ & $0.98 \pm 0.06^{*}$ & $1.06 \pm 0.05^{*}$ \\
Gender, M/F & $61 / 71$ & $52 / 65$ & $54 / 58$ \\
SBP, mm Hg & $127 \pm 9$ & $132 \pm 8^{\#}$ & $137 \pm 8$ \\
DBP, mm Hg & $77 \pm 7$ & $75 \pm 7$ & $78 \pm 9$ \\
HR, bmp & $83 \pm 14$ & $81 \pm 11$ & $86 \pm 11$ \\
BG, mmol/l & $4.7 \pm 0.6$ & $5.0 \pm 0.6$ & $5.1 \pm 0.8$ \\
Tchol, mmol/l & $4.3 \pm 0.6$ & $4.4 \pm 0.7$ & $4.7 \pm 0.6$ \\
TG, mmol/l & $1.48 \pm 0.76$ & $1.41 \pm 0.83$ & $1.7 \pm 1.54$ \\
LDL, mmol/l & $2.43 \pm 0.64$ & $2.76 \pm 0.72$ & $2.98 \pm 0.65$ \\
HDL, mmol/l & $1.03 \pm 0.26$ & $1.08 \pm 0.27$ & $1.00 \pm 0.29$ \\
Cr, $\mu \mathrm{mol} / \mathrm{l}$ & $67 \pm 13$ & $73 \pm 15$ & $74 \pm 18$ \\
\hline
\end{tabular}

G1 = Normal weight and overweight group; G2 = mild and moderate obesity group; G3 = severe obesity group.

Compared with G1: G2, *p $<0.01$, ${ }^{*} \mathrm{p}<0.05$.

Compared with G2: G3, *p $<0.01$.

\begin{tabular}{llll}
\hline & G1 $(\mathrm{n}=132)$ & $\mathrm{G} 2(\mathrm{n}=117)$ & $\mathrm{G} 3(\mathrm{n}=112)$ \\
\hline LVEDD, mm & $47.4 \pm 4.9$ & $54.0 \pm 5.0^{*}$ & $60.0 \pm 5.2^{*}$ \\
IVS, mm & $9.3 \pm 1.2$ & $11.5 \pm 1.5^{*}$ & $12.1 \pm 1.4$ \\
LVPW, mm & $9.2 \pm 1.2$ & $11.4 \pm 1.4^{*}$ & $12.0 \pm 1.3$ \\
LV mass, g & $193 \pm 63$ & $319 \pm 98^{*}$ & $403 \pm 112^{*}$ \\
LVMI, g/m ${ }^{2.7}$ & $103 \pm 24$ & $142 \pm 39^{*}$ & $143 \pm 31$ \\
LAD, mm & $33.4 \pm 3.4$ & $37.2 \pm 4.6^{*}$ & $40.6 \pm 5.5^{\#}$ \\
LVEF, \% & $63.6 \pm 4.5$ & $61.6 \pm 6.8$ & $57.3 \pm 5.5^{\#}$ \\
E/A (mitral) & $1.41 \pm 0.42$ & $1.15 \pm 0.30^{*}$ & $1.16 \pm 0.30$ \\
E/e' (mitral) & $8.2 \pm 1.9$ & $9.5 \pm 2.3^{*}$ & $11.4 \pm 2.9^{\#}$
\end{tabular}

G1 = Normal weight and overweight group; G2 = mild and moderate obesity group; G3 = severe obesity group.

Compared with G1: G2, *p $<0.01$, ${ }^{\#} \mathrm{p}<0.05$.

Compared with G2: G3, *p $<0.01$.

(G3). Because age influences LV structure and function, the age distribution of each group did not differ. The clinical characteristics of each group are shown in table 1. Obese patients had higher systolic blood pressure (SBP) than the normal/overweight patients. Except for BMI and WHR, there were no significant differences in other characteristics among these groups, including age, gender, diastolic blood pressure (DBP), heart rate (HR), lipid profile, $\mathrm{Cr}$, and BG.

\section{Structure and Function}

Comparisons of echocardiographic parameters are shown in table 2. There was a significant difference in cardiac structure parameters, including LVEDD, IVS, LVPW, LV mass, LVMI and LAD, among the three groups. Compared to the normal/overweight group (G1), mildly/ moderately obese patients (G2) had a larger heart cavity, thicker IVS and LVPW as well as larger LV mass and LVMI, but there was no difference in LVEF, which indicated LV hypertrophy in the mildly/moderately obese patients. Severely obese patients had a larger heart 
Sun et al.: Left Ventricular Hypertrophy and Asymptomatic Cardiac Function

Impairment in Chinese Patients with Simple Obesity using Echocardiography

Table 3. Echocardiographic characteristics in gender and age subgroups and G1 subgroup

\begin{tabular}{|c|c|c|c|c|c|c|}
\hline & \multicolumn{2}{|l|}{ Age group } & \multicolumn{2}{|c|}{ Gender group } & \multicolumn{2}{|l|}{ G1 group } \\
\hline & $\begin{array}{l}G-Y \\
(n=223)\end{array}$ & $\begin{array}{l}\text { G-O } \\
(n=138)\end{array}$ & $\begin{array}{l}\text { G-F } \\
(n=194)\end{array}$ & $\begin{array}{l}\text { G-M } \\
(n=167)\end{array}$ & $\begin{array}{l}\text { G1-1 } \\
(\mathrm{n}=74)\end{array}$ & $\begin{array}{l}\text { G1-2 } \\
(n=58)\end{array}$ \\
\hline Age, years & $27.8 \pm 4.6$ & $43.1 \pm 5.5^{*}$ & $32.9 \pm 8.9$ & $33.5 \pm 9.0$ & $28.9 \pm 6.3$ & $35.5 \pm 8.9^{\#}$ \\
\hline BMI, $\mathrm{kg} / \mathrm{m}^{2}$ & $28.9 \pm 10.6$ & $33.4 \pm 11.6^{\#}$ & $29.3 \pm 11.6$ & $31.7 \pm 10.6^{\#}$ & $20.1 \pm 2.6$ & $25.7 \pm 2.5^{*}$ \\
\hline WHR & $0.90 \pm 0.12$ & $0.95 \pm 0.10^{*}$ & $0.90 \pm 0.13$ & $0.94 \pm 0.09^{\#}$ & $0.82 \pm 0.06$ & $0.90 \pm 0.07^{*}$ \\
\hline LVEDD, mm & $49.9 \pm 6.7$ & $54.0 \pm 6.8^{*}$ & $49.1 \pm 6.6$ & $53.8 \pm 6.6^{*}$ & $46.1 \pm 4.4$ & $51.8 \pm 4.1^{*}$ \\
\hline IVS, mm & $9.8 \pm 1.6$ & $11.3 \pm 1.7^{*}$ & $9.9 \pm 1.8$ & $10.9 \pm 1.7^{*}$ & $8.8 \pm 0.8$ & $10.8 \pm 1.1^{*}$ \\
\hline LVPW, mm & $9.8 \pm 1.6$ & $11.2 \pm 1.7^{*}$ & $9.8 \pm 1.7$ & $10.8 \pm 1.7^{*}$ & $8.8 \pm 0.8$ & $10.7 \pm 1.0^{*}$ \\
\hline LV mass, g & $233 \pm 107$ & $316 \pm 120^{*}$ & $223 \pm 108$ & $299 \pm 119^{*}$ & $170 \pm 42$ & $269 \pm 62^{*}$ \\
\hline LVMI, g/m $\mathrm{m}^{2.7}$ & $109 \pm 27$ & $141 \pm 39 *$ & $113 \pm 33$ & $128 \pm 36^{*}$ & $96 \pm 18$ & $127 \pm 26^{*}$ \\
\hline $\mathrm{LAD}, \mathrm{mm}$ & $33.1 \pm 4.5$ & $37.4 \pm 5.8^{*}$ & $33.3 \pm 4.8$ & $36.0 \pm 5.7^{*}$ & $30.4 \pm 2.9$ & $35.4 \pm 2.6^{*}$ \\
\hline LVEF, \% & $62.9 \pm 6.0$ & $60.2 \pm 5.0^{*}$ & $62.8 \pm 5.9$ & $61.1 \pm 5.7$ & $63.8 \pm 4.4$ & $62.9 \pm 4.7$ \\
\hline E/A (mitral) & $1.42 \pm 0.38$ & $1.07 \pm 0.29 *$ & $1.37 \pm 0.40$ & $1.22 \pm 0.37$ & $1.46 \pm 0.38$ & $1.22 \pm 0.48$ \\
\hline $\mathrm{E} / \mathrm{e}^{\prime}$ (mitral) & $9.7 \pm 2.5$ & $9.2 \pm 2.4$ & $9.0 \pm 2.5$ & $9.3 \pm 2.4$ & $9.0 \pm 2.5$ & $9.3 \pm 2.4$ \\
\hline
\end{tabular}

$\mathrm{G}-\mathrm{Y}=$ Young subgroup (age $\leq 35$ years); $\mathrm{G}-\mathrm{O}=$ middle/old subgroup (age $>35$ years); $\mathrm{G}-\mathrm{F}=$ female subgroup; G-M = male subgroup; G1-1 = normal-weight group; G1-2 = overweight group.

Within group comparisons: ${ }^{*} \mathrm{p}<0.01,{ }^{*} \mathrm{p}<0.05$.

cavity, larger LV mass, and lower LVEF than the mildly/moderately obese group (G2), but there was no difference in IVS, LVPW and LVMI, which suggested that LV enlargement was accompanied by LV systolic function impairment in the severe obesity group.

$\mathrm{LV}$ diastolic function was evaluated using the $\mathrm{E} / \mathrm{A}$ and $\mathrm{E} / \mathrm{e}^{\prime}$ ratios. The $\mathrm{E} / \mathrm{A}$ ratio refers to the ratio of E-wave mitral velocity to A-wave mitral velocity at the level of the mitral valve leaflet tips. Impaired relaxation was detected by a reduction in $\mathrm{E} / \mathrm{A}$ ratio. The $\mathrm{E} / \mathrm{A}$ ratio was significantly higher in the normal/overweight group than in the mildly/moderate obesity group, but there were no significant differences between the mildly/moderate obesity and severe obesity groups. Increased filling pressure was considered to be present in patients with an increased $\mathrm{E} / \mathrm{e}^{\prime}$ ratio, which became progressively higher from the overweight to the severe obesity groups; thus, diastolic dysfunction was significantly higher in the severe obesity group than in the overweight/obese groups.

In the subgroup analyses, we divided the participants according to age and gender. As shown in table 3, there were higher BMI and WHR, larger heart cavity, thicker myocardial wall, and lower LVEF and E/A in the middle-aged/old subgroup (age > 35 years) than in the young subgroup (age $<35$ years). We also found that males had higher BMI and WHR, larger LVEDD and LAD, thicker septum and posterior wall, and larger LV mass and LVMI than females. To understand whether being overweight affects cardiac structure and function, the G1 group was divided into an overweight subgroup and a normal subgroup. The overweight subgroup had a larger heart and thicker ventricular wall than the normal subgroup. However, there were no significant differences in functional characteristics, including LVEF, E/A ratio and $\mathrm{E} / \mathrm{e}^{\prime}$ ratio, among these subgroups.

\section{Interrelations}

Multivariate correlation and logistic regression analysis demonstrated the association of BMI or WHR with clinical and echocardiographic parameters (table 4). A series of stepwise multiple regression models were developed to identify independent associations of BMI or WHR. The set of variables tested in these models included laboratory and echocardiographic parameters. The variables were put into the stepwise models in order of descending signifi- 
Table 4. The association of BMI and WHR with clinical and echocardiographic parameter
Sun et al.: Left Ventricular Hypertrophy and Asymptomatic Cardiac Function Impairment in Chinese Patients with Simple Obesity using Echocardiography

\begin{tabular}{|c|c|c|c|c|c|c|}
\hline & \multicolumn{3}{|c|}{$\begin{array}{l}\text { BMI } \\
\left(R^{2}=0.891 ; p<0.000\right)\end{array}$} & \multicolumn{3}{|c|}{$\begin{array}{l}\text { WHR } \\
\left(\mathrm{R}^{2}=0.732 ; \mathrm{p}<0.000\right)\end{array}$} \\
\hline & Coeff & SE & $\mathrm{p}$ & Coeff & SE & $\mathrm{p}$ \\
\hline Age & 0.064 & 0.056 & 0.150 & -0.079 & 0.001 & 0.260 \\
\hline SBP & 0.091 & 0.050 & 0.032 & 0.088 & 0.001 & 0.184 \\
\hline DBP & -0.048 & 0.057 & 0.205 & -0.167 & 0.001 & 0.006 \\
\hline $\mathrm{BG}$ & 0.004 & 0.585 & 0.918 & 0.029 & 0.009 & 0.596 \\
\hline HR & 0.019 & 0.030 & 0.592 & 0.062 & 0.000 & 0.269 \\
\hline $\mathrm{Cr}$ & 0.052 & 0.026 & 0.131 & -0.008 & 0.000 & 0.884 \\
\hline Tchol & -0.004 & 0.565 & 0.900 & -0.006 & 0.009 & 0.912 \\
\hline TG & -0.032 & 0.385 & 0.342 & 0.084 & 0.006 & 0.110 \\
\hline LDL & 0.040 & 0.548 & 0.248 & 0.112 & 0.009 & 0.039 \\
\hline HDL & -0.037 & 1.430 & 0.279 & -0.072 & 0.023 & 0.179 \\
\hline LVEF & $\begin{array}{c}-0.058 \\
0.309\end{array}$ & 0.070 & 0.112 & $\begin{array}{l}-0.034 \\
0.648\end{array}$ & 0.001 & 0.554 \\
\hline LVEDD & 0.309 & 0.186 & 0.009 & 0.648 & 0.003 & 0.001 \\
\hline LAD & 0.058 & 0.146 & 0.414 & 0.176 & 0.002 & 0.114 \\
\hline IVS & -0.260 & 1.578 & 0.314 & -0.510 & 0.025 & 0.208 \\
\hline LVPW & 0.725 & 1.559 & 0.004 & 1.460 & 0.025 & 0.000 \\
\hline LV mass & 0.917 & 0.019 & 0.000 & -0.641 & 0.000 & 0.046 \\
\hline LVMI & -1.096 & 0.031 & 0.000 & -0.406 & 0.000 & 0.009 \\
\hline E/A (mitral) & 0.012 & 1.190 & 0.771 & -0.097 & 0.019 & 0.140 \\
\hline $\mathrm{E} / \mathrm{e}^{\prime}$ (mitral) & 0.082 & 0.171 & 0.034 & 0.007 & 0.003 & 0.911 \\
\hline
\end{tabular}

Coeff = Sample estimate of the regression parameter; $\mathrm{SE}=$ standard error of the estimated regression coefficient.

cance in the univariate analyses. The independent determinants were SBP/DBP, LDL-C, LVEDD, LVPW, LV mass, LVMI, and E/e' ratio (mitral).

We also studied the associations of echocardiographic parameters with clinical and laboratory characteristics using Pearson's test (r) (table 5). Significant correlations were revealed between BMI and LVEDD, LVPW, LV mass, LVMI or E/e' ratio and between WHR and LVEDD, LVPW, LV mass or LVMI. WHR was found to be directly correlated with LDL-C and DBP, and BMI was correlated with SBP but not with $\mathrm{HR}, \mathrm{BP}, \mathrm{Cr}$, Tchol, or TG. Additionally, we found that age was significantly positively associated with some echocardiographic parameters, including LAD ( $\mathrm{r}=0.366, \mathrm{p}<0.01)$, LVPW $(\mathrm{r}=0.347, \mathrm{p}<0.01)$, and IVS $(\mathrm{r}=0.351, \mathrm{p}<0.01)$, and was negatively associated with $\mathrm{E} / \mathrm{A}$ ratio $(\mathrm{r}=-0.47, \mathrm{p}<0.01)$. There was no relationship between other characteristics and the echocardiographic parameters.

\section{Discussion}

In the present study, we have compared cardiac structure and function in patients with various degrees of obesity using echocardiography and also evaluated the relationship between clinical characteristics and echocardiographic parameters. The main clinical findings were the following: i) Patients with mild and moderate obesity demonstrated LV hypertrophy and enlargement. ii) Patients with severe obesity showed not only LV hypertrophy but also asymptomatic cardiac function impairment. iii) These cardiac structural and functional changes were independently determined by BMI, WHR, and age, and not by HR, BP, BG, Cr, Tchol, or TG.

There has been a significant increase in the incidence of obesity, followed by an increased prevalence of hypertension and heart failure, in China during the past decades [15]. Obesity, 
Sun et al.: Left Ventricular Hypertrophy and Asymptomatic Cardiac Function

Impairment in Chinese Patients with Simple Obesity using Echocardiography

Table 5. Correlations of clinical and echocardiographic characteristics

\begin{tabular}{|c|c|c|c|c|c|c|c|c|c|c|c|c|c|c|c|c|c|c|}
\hline & \multicolumn{2}{|l|}{ BMI } & \multicolumn{2}{|c|}{ LVEDD } & \multicolumn{2}{|l|}{ LVPW } & \multicolumn{2}{|c|}{ LV mass } & \multicolumn{2}{|l|}{ LVMI } & \multicolumn{2}{|l|}{ LAD } & \multicolumn{2}{|l|}{ LVEF } & \multicolumn{2}{|l|}{$\mathrm{E} / \mathrm{A}$} & \multicolumn{2}{|l|}{$\mathrm{E} / \mathrm{e}^{\prime}$} \\
\hline & $r$ & $\mathrm{p}$ & $r$ & $\mathrm{p}$ & $\mathrm{r}$ & $\mathrm{p}$ & $\mathrm{r}$ & $\mathrm{p}$ & $\mathrm{r}$ & $\mathrm{p}$ & $\mathrm{r}$ & $\mathrm{p}$ & $r$ & $\mathrm{p}$ & $\mathrm{r}$ & $\mathrm{p}$ & $\mathrm{r}$ & $\mathrm{p}$ \\
\hline BMI & / & / & 0.78 & 0.00 & 0.76 & 0.00 & 0.84 & 0.00 & 0.56 & 0.00 & 0.73 & 0.00 & -0.39 & 0.00 & -0.34 & 0.00 & 0.51 & 0.00 \\
\hline WHR & 0.84 & 0.00 & 0.70 & 0.00 & 0.75 & 0.00 & 0.72 & 0.00 & 0.58 & 0.00 & 0.69 & 0.00 & -0.36 & 0.00 & -0.38 & 0.00 & 0.44 & 0.00 \\
\hline Age & 0.10 & 0.23 & 0.24 & 0.01 & 0.35 & 0.00 & 0.30 & 0.00 & 0.44 & 0.00 & 0.37 & 0.00 & -0.14 & 0.10 & -0.47 & 0.00 & -0.04 & 0.63 \\
\hline SBP & 0.35 & 0.02 & 0.23 & 0.06 & 0.46 & 0.00 & 0.50 & 0.00 & 0.43 & 0.00 & 0.32 & 0.03 & -0.08 & 0.34 & -0.18 & 0.04 & 0.23 & 0.01 \\
\hline DBP & 0.11 & 0.21 & 0.20 & 0.10 & 0.13 & 0.13 & 0.11 & 0.20 & 0.09 & 0.29 & 0.14 & 0.18 & -0.03 & 0.73 & -0.09 & 0.30 & 0.08 & 0.22 \\
\hline HR & 0.05 & 0.56 & 0.08 & 0.36 & 0.05 & 0.56 & 0.02 & 0.74 & 0.12 & 0.17 & 0.10 & 0.26 & -0.12 & 0.37 & -0.09 & 0.32 & 0.04 & 0.70 \\
\hline BS & 0.10 & 0.06 & 0.08 & 0.16 & 0.10 & 0.09 & 0.19 & 0.11 & 0.21 & 0.08 & 0.14 & 0.26 & -0.15 & 0.08 & -0.13 & 0.15 & 0.08 & 0.34 \\
\hline Tchol & 0.09 & 0.07 & 0.18 & 0.13 & 0.19 & 0.30 & 0.06 & 0.50 & 0.09 & 0.48 & 0.19 & 0.12 & -0.09 & 0.29 & -0.06 & 0.32 & 0.13 & 0.15 \\
\hline $\mathrm{TG}$ & 0.02 & 0.84 & 0.06 & 0.64 & 0.12 & 0.81 & 0.13 & 0.28 & 0.03 & 0.74 & 0.11 & 0.38 & -0.16 & 0.08 & 0.07 & 0.58 & 0.06 & 0.63 \\
\hline LDL & 0.06 & 0.48 & 0.13 & 0.11 & 0.16 & 0.10 & 0.11 & 0.30 & 0.19 & 0.24 & 0.23 & 0.08 & -0.04 & 0.68 & -0.24 & 0.26 & 0.16 & 0.19 \\
\hline HDL & -0.09 & 0.31 & -0.05 & 0.56 & -0.05 & 0.60 & -0.06 & 0.46 & -0.04 & 0.35 & -0.07 & 0.58 & 0.13 & 0.19 & 0.12 & 0.23 & -0.09 & 0.46 \\
\hline $\mathrm{Cr}$ & 0.06 & 0.65 & 0.10 & 0.26 & 0.06 & 0.58 & 0.10 & 0.49 & 0.02 & 0.80 & 0.09 & 0.30 & -0.05 & 0.60 & -0.04 & 0.65 & -0.07 & 0.39 \\
\hline
\end{tabular}

as a risk factor for diabetes, has gained recognition in China [16]. However, there is little concern about the relationship between simple obesity and heart disease. Therefore, we evaluated clinical and echocardiographic characteristics in patients with simple obesity and analyzed changes in cardiac structure and function. Our results showed that obese patients were more likely to have LV enlargement and hypertrophy, which was directly positively correlated with BMI. Because there were no differences in other clinical characteristics, including age, HR, BP, BG, Cr, Tchol, TG, LDL-C and HDL-C, among the various obese groups, simple obesity contributes to the development of LV hypertrophy and enlargement.

LVEF, as a recognized predictor of LV systolic function decline, was lowest in the severely obese group, although all these data were in the normal range (50-80\%). Diastolic dysfunction was classified as mild if $\mathrm{E} / \mathrm{A}<0.7$ (impaired relaxation), moderate if $\mathrm{E} / \mathrm{A}>0.7$ and $<1.5$ (pseudo-normalized pattern), and severe if $\mathrm{E} / \mathrm{A}>1.5$ (restrictive pattern) [16]. To clarify the role of obesity in $\mathrm{LV}$ diastolic function, we analyzed the $\mathrm{E} / \mathrm{A}$ and $\mathrm{E} / \mathrm{e}^{\prime}$ ratios of the three groups and found that the mildly/moderate obesity group had a lower E/A ratio and higher E/e' ratio than the normal/overweight group. Additionally, the severe obesity group had a higher E/ $\mathrm{e}^{\prime}$ ratio but no change in E/A ratio compared to the mildly/moderate obesity group, which may be due to the pseudo-normalized pattern of the E/A ratio in the severe obesity group. Decreased E/A and increased E/e' ratios indicated increased filling pressure, which suggested the presence of diastolic dysfunction. These data reveal that severe obesity impairs not only $\mathrm{LV}$ systolic function but also LV diastolic function.

The key observation in the present study was that alterations in the cardiac structure and function of severely obese patients were different from those of mildly/moderately obese patients. Compared to the normal/overweight group, there were thicker IVS and LVPW and higher LVMI in the mildly/moderately obese group but not in the severely obese group (table 2). However, there was a significantly lower LVEF in the severely obese group, but not in the mildly/moderately obese group. The mildly/moderately obese patients showed LV hypertrophy and very early diastolic dysfunction, but severely obese patients showed LV enlargement and systolic and diastolic function impairment. All these data suggest that obesity might result in myocardial remodeling, accompanied by LV function disturbances that increase with the degree of obesity. Additionally, LV mass and LVMI are the absolute and relative weights of the heart, respectively, and LVMI is more accurate for evaluating myocardial remodeling $[17,18]$. As shown in table 2 , severely obese patients had larger LV mass, but not 
LVMI, than the mildly/moderately obese patients, which indicates that the degree of myocardial remodeling was not different between the two groups, despite the heavier hearts of the severely obese patients.

The results of the correlation analysis further confirmed the relationship between obesity and heart structure. A significant positive correlation was observed between BMI or WHR and echocardiographic parameters, including LVEDD, LVPW, LV mass and LVMI, which suggested that BMI and WHR are independent determinants of LV hypertrophy. Increasing BMI was associated with increasing $\mathrm{E} / \mathrm{e}^{\prime}$ ratio, which also indicates that patients with severe obesity exhibited asymptomatic LV diastolic function impairment. We also evaluated the association of echocardiographic parameters with other clinical characteristics, including HR, BP, BG, Cr, Tchol and TG, and did not find any significant relationships between them. All these data suggest that simple obesity is an independent risk factor for LV hypertrophy and dysfunction. Therefore, weight loss is necessary for most obese patients.

Many scientists have studied the relationships between obesity and heart diseases with a special emphasis on potential mechanisms. Eschalier et al. [19] reported that early cardiac structural remodeling, fibrosis and diastolic dysfunction were detectable in healthy but obese adolescents. Linz et al. [20] found increased LV fibrosis with increased myocyte diameters and an impaired LV ejection fraction in an obese, spontaneously hypertensive rat model. However, the pathophysiology of obesity-induced LV remodeling and dysfunction is not clear. Increased blood volume and heart overload may be involved in the pathogenesis of myocardial hypertrophy [20]. Cardiac overload is associated with increased activation of the renin-angiotensin-aldosterone system (RAAS) [20]. Therefore, increased RAAS activation may stimulate myocardial hypertrophy and fibrosis, leading to LV systolic and diastolic dysfunction. The molecular mechanisms of the effects of obesity on cardiac contractility were studied in previous experimental studies, including oxidative stress [21, 22], calcium mishandling [22], high-sensitivity C-reactive protein [23], insulin resistance [22, 24], and arterial stiffness [25].

We have demonstrated that age was also independently associated with LV hypertrophy and dysfunction. Middle-aged/old patients had larger heart cavities, thicker myocardial walls, and lower LVEF and E/A ratios than young patients. Age was also significantly positively associated with LV hypertrophy and very early LV diastolic function, but not with BMI. Therefore, age was also a factor that mediated LV hypertrophy and diastolic dysfunction but was independent of BMI. We also analyzed the effect of gender on cardiac structure and function and found that male hearts were always larger than female hearts, but the function was not different, and there was no difference in overweight individuals (table 3).

Consistent with the results of many studies on obesity and hypertension [26-28], we found that SBP was increased in obese patients and positively associated with BMI, LVEDD as well as myocardial thickness and function. Obesity can lead to hypertension, which is closely related to heart structure and function. In fact, obesity, hypertension, and LV hypertrophy are inseparable. Obesity is also an independent risk factor for hypertension. The mechanisms underlying obesity-associated hypertension have not yet been clearly understood. There are some leading hypothetical mechanisms to explain obesity-associated hypertension, including activation of the RAAS, adipose tissue and endothelial dysfunction [26-28].

\section{Limitations}

Given our strict exclusion criteria, this study is homocentric and cross-sectional, and the studied population size was limited. The proof of clinically documented preexisting cardiovascular disease in our study participants was only based on the noninvasive strategy used in the study protocol; so we could not clearly ascertain the absence of coronary artery disease. However, this factor seems unavoidable because an angiographic verification would not be 
justified in asymptomatic individuals for ethical reasons. As numerous comparisons were performed with no adjustment for multiple testing, the increased risk of type I errors should be acknowledged.

\section{Conclusion}

LV hypertrophy and enlargement were often observed in the mildly/moderately obese group, and asymptomatic LV function impairment was observed particularly in the severely obese group. An increased BMI and WHR were directly correlated with LV hypertrophy factors such as LVEDD, LVPW, LV mass, and LVMI. Additionally, age had a positive relationship with LV hypertrophy and diastolic dysfunction. These observations indicate that simple severe obesity is an independent risk factor for cardiac dilatation, hypertrophy as well as asymptomatic LV systolic and diastolic function impairment.

\section{Author Contributions}

Conceived and designed the experiments: TS, HZ, XY. Performed the experiments: TS, XJ, ZL. Analyzed the data: TS, XY. Wrote the first draft of the manuscript: TS. Contributed to the writing of the manuscript: TS, HZ. All authors reviewed and approved the final manuscript.

\section{Acknowledgments}

This work was supported by the National Natural Science Foundation (No. 81201116) and Shanghai Administration of traditional Chinese medicine subject (No.cc-3-3006).

\section{Disclosure Statement}

The authors declare that they have no conflicts of interest.

\section{References}

1 Tian X, Zhao G, Li Y, Wang L, Shi Y: Overweight and obesity difference of Chinese population between different urbanization levels. J Rural Health 2014;30:101-112.

2 von Deneen KM, Wei Q, Tian J, Liu Y: Obesity in China: what are the causes? Curr Pharm Des 2011;17:11321139.

-3 Jin MJ, Chen BB, Mao YY, Zhu YM, Yu YX: Prevalence of overweight and obesity and their associations with socioeconomic status in a rural Han Chinese adult population. PloS One 2013;8:e79946.

4 Dalzill C, Nigam A, Juneau M, Guilbeault V, Latour E, Mauriège P, Gayda M: Intensive lifestyle intervention improves cardiometabolic and exercise parameters in metabolically healthy obese and metabolically unhealthy obese individuals. Can J Cardiol 2014;30:434-440.

-5 Wierzbowska-Drabik K, Chrzanowski L, Kapusta A, Uznańska-Loch B, Płońska E, Krzemińska-Pakuła M, Kurpesa M, Rechciński T, Trzos E, Kasprzak JD: Severe obesity impairs systolic and diastolic heart function the significance of pulsed tissue Doppler, strain, and strain rate parameters. Echocardiography 2013;30:904911.

6 Sullivan PW, Ghushchyan VH, Ben-Joseph R: The impact of obesity on diabetes, hyperlipidemia and hypertension in the United States. Qual Life Res 2008;17:1063-1071.

7 DeMarco VG, Aroor AR, Sowers JR: The pathophysiology of hypertension in patients with obesity. Nat Rev Endocrinol 2014;10:364-376.

-8 Dolan LC, Potter SM, Burdock GA: Evidence-based review on the effect of normal dietary consumption of fructose on development of hyperlipidemia and obesity in healthy, normal weight individuals. Crit Rev Food Sci Nutr 2010;50:53-84.

-9 Lee BC, Lee J: Cellular and molecular players in adipose tissue inflammation in the development of obesityinduced insulin resistance. Biochim Biophys Acta 2014;1842:446-462. 
Sun et al.: Left Ventricular Hypertrophy and Asymptomatic Cardiac Function

Impairment in Chinese Patients with Simple Obesity using Echocardiography

10 Du Bois D, Du Bois EF: A formula to estimate the approximate surface area if height and weight be known. Nutrition 1989;5:301-311.

11 Wang SK, Ma W, Wang S, Yi XR, Jia HY, Xue F: Obesity and its relationship with hypertension among adults 50 years and older in Jinan, China. PLoS One 2014; 9:e114424.

12 Devereux RB, Reichek N: Echocardiographic determination of left ventricular mass in man. Anatomic validation of the method. Circulation.1977;55:613-8.

13 Lapu-Bula R, Quarshie A, Lyn D, Oduwole A, Pack C, Morgan J, Nkemdiche S, Igho-Pemu P, Onwuanyi A, Li R, Ofili E. The $894 \mathrm{~T}$ allele of endothelial nitric oxide synthase gene is related to left ventricular mass in African Americans with high-normal blood pressure. J Natl Med Assoc 2005;97:197-205.

14 Kilicaslan B, Ozdogan O, Aydin M, Dursun H, Susam I: Increased epicardial fat thickness is associated with cardiac functional changes in healthy women. Tohoku J Exp Med 2012;228:119-124.

15 Lu J, Bi Y, Wang T, Wang W, Mu Y, Zhao J: The relationship between insulin-sensitive obesity and cardiovascular diseases in a Chinese population: results of the REACTION study. Int J Cardiol 2014;172:388-394.

16 Kossaify A, Nicolas N: Impact of overweight and obesity on left ventricular diastolic function and value of tissue Doppler echocardiography. Clin Med Insights Cardiol 2013;7:43-50.

17 Krishnan R, Becker RJ, Beighley LM, López-Candales A: Impact of body mass index on markers of left ventricular thickness and mass calculation: results of a pilot analysis. Echocardiography 2005;22:203-210.

18 Powell BD, Redfield MM, Bybee KA, Freeman WK, Rihal CS: Association of obesity with left ventricular remodeling and diastolic dysfunction in patients without coronary artery disease. Am J Cardiol 2006;98:116-120.

19 Eschalier R, Rossignol P, Kearney-Schwartz A, Adamopoulos C, Karatzidou K, Fay R, Mandry D, Marie PY, Zannad F: Features of cardiac remodeling, associated with blood pressure and fibrosis biomarkers, are frequent in subjects with abdominal obesity. Hypertension 2014;63:740-746.

-20 Linz D, Hohl M, Mahfoud F, Reil JC, Linz W, Hübschle T, Juretschke HP, Neumann-Häflin C, Rütten H, Böhm M: Cardiac remodeling and myocardial dysfunction in obese spontaneously hypertensive rats. J Transl Med 2012; 10:187.

21 Noeman SA, Hamooda HE, Baalash AA: Biochemical study of oxidative stress markers in the liver, kidney and heart of high fat diet induced obesity in rats. Diabetol Metab Syndr 2011;3:17.

22 Carvajal K, Balderas-Villalobos J, Bello-Sanchez MD, Phillips-Farfán B, Molina-Muñoz T, Aldana-Quintero H, Gómez-Viquez NL: $\mathrm{Ca}(2+)$ mishandling and cardiac dysfunction in obesity and insulin resistance: role of oxidative stress. Cell Calcium 2014;56:408-415.

-23 Indulekha K, Surendar J, Anjana RM, Geetha L, Gokulakrishnan K, Pradeepa R,Mohan V: Metabolic obesity, adipocytokines, and inflammatory markers in Asian Indians - CURES-124. Diabetes Technol Ther 2015;17: 134-141.

24 Antonini-Canterin F, Mateescu AD, Vriz O, La Carrubba S, Di Bello V, Carerj S, Zito C, Sparacino L, Marzano B, Usurelu C, Ticulescu R, Ginghină C, Nicolosi GL, Popescu BA: Cardiac structure and function and insulin resistance in morbidly obese patients: does superobesity play an additional role? Cardiology 2014;127:144-151.

25 Alvey NJ, Pedley A, Rosenquist KJ, Massaro JM, O’Donnell CJ, Hoffmann U, Fox CS: Association of fat density with subclinical atherosclerosis. J Am Heart Assoc 2014;3:e000788.

26 DeMarco VG, Aroor AR, Sowers JR: The pathophysiology of hypertension in patients with obesity. Nat Rev Endocrinol 2014;10:364-376.

-27 Vaněčková I, Maletínská L, Behuliak M, Nagelová V, Zicha J, Kuneš J: Obesity-related hypertension: possible pathophysiological mechanisms. J Endocrinol 2014;223:R63-78.

28 Kang YS: Obesity associated hypertension: new insights into mechanism. Electrolyte Blood Press 2013;11: 46-52. 